\title{
Liste des thèses d'anthropologie soutenues en 2015
}

\section{Université d'Aix-Marseille}

Pierre Frémondière : Stratégie de subsistance en Italie à l'âge du Bronze : une approche isotopique de l'alimentation humaine.

Justin Michel : Intérêts anthropologiques, médicolégaux et cliniques des constructions tridimensionnelles.

\section{Université d'Aix-Marseille et cotutelle université de Firenze}

Alessandra Varalli : Stratégie de subsistance en Italie à l'âge du Bronze : une approche isotopique de l'alimentation humaine.

\section{Université Bordeaux-I}

Arwa Kharobi : Approche archéo-anthropologique sur les tombes $d u$ Chalcolithique à l'âge du Bronze moyen de Tell Hamoukar et Tell Mozan au nord-est de la Syrie: taphonomie, évolution des pratiques funéraires.

Caroline Laforest: La sépulture collective $163 d$ de la nécropole nord de Hiérapolis (Phrygie, Turquie, $I^{e r}$ siècle-VI ${ }^{e}$ siècle de notre ère) : biologie, gestes et pratiques funéraires.

Mélie Le Roy: Les pratiques funéraires réservées aux enfants au cours de la période du Néolithique (VI ${ }^{e}-I I I^{e}$ millénaire av. J.-C.) en France.

Kévin Salesse : Étude bioarchéologique d'une population ancienne reliée à une crise de mortalité : les inhumés de la catacombe des Saints-Pierre-et-Marcelin à Rome

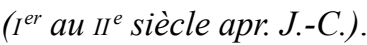

\section{Université de Poitiers}

Anne Le Maitre: Locomotion et morphologie du labyrinthe osseux chez les hominö̈des actuels, application à Sahelanthropus tchadensis dit "Toumaï» (Hominidae, $7 \mathrm{Ma}$, Tchad).

\section{Université de Toulouse-III}

Amélie Beaudet : Caractérisation des structures craniodentaires internes des cercopithécoïdes et étude diachronique de leurs variations morphologiques dans la séquence Plio-Pléistocène sud-africaine.

Marie Faruch: Étude radiologique du dimorphisme sexuel de l'os coxal immature.

Cesar Fortes-Lima : Approche génétique de l'origine des communautés afro-américaines en Amérique du Sud.

Mejdoubi : Étude du développement de l'oreille interne en TDM chez le foetus.

\section{Muséum national d'histoire naturelle}

Marie Cibot: Le chimpanzé face à l'anthropisation de son habitat: conséquences sanitaires, comportementales, fonctionnelles et sociales.

Sandrine Dupé: Séparer les moustiques des humains. Co-production d'un nouvel ordre socio-naturel en contexte post-colonial (Île de la Réunion).

Emeline Lechaux : Tisser le fil de la mémoire : contribution à l'histoire des répertoires musicaux des cérémonies de «bwétè » chez les Mitsogo du Gabon.

Elise Lucotte: Détection de processus sexuellement antagonistes dans le génome humain.

Maho Sebiane : L'Invisible : esclavage, sawâhili et possession dans le complexe rituel leiwah d'Arabie orientale (Sultanat d'Oman - Émirats Arabes Unis).

Agnès Sjostrand : Origins and Adaptation in Humans: a Case Study of Taste and Lifestyle. 